\title{
RINGS OF CONTINUOUS FUNCTIONS AND TOTALLY INTEGRALLY CLOSED RINGS ${ }^{1}$
}

\author{
M. HOCHSTER
}

0. Introduction. We shall prove that the ring of continuous complex-valued functions on a completely regular Hausdorff space is totally integrally closed iff the space is extremally disconnected.

$X$ always denotes a completely regular Hausdorff space. $\mathbf{R}$ is the field of real numbers, $\mathbf{C}$ of complex numbers. $C(X)$ denotes the ring of all continuous $\mathrm{R}$-valued functions on $X, C^{*}(X)$ the subring of bounded functions in $C(X), i=\sqrt{ }(-1)$. Then $C(X)[i]$ (respectively, $\left.C^{*}(X)[i]\right)$ is the ring of continuous (respectively, bounded continuous) $\mathrm{C}$-valued functions of $X$.

A ring $A$ (ring $\equiv$ commutative ring with 1 ) is totally integrally closed if for every integral extension $h: B \rightarrow C$, the induced map $h^{*}: \operatorname{Hom}(C, A)$ $\rightarrow \operatorname{Hom}(B, A)$ is surjective. See [1] and [4] for a detailed treatment. By [4, Theorem 1 and Proposition 5], $A$ is totally integrally closed iff the following four conditions hold:

(1) $A$ is reduced,

(2) every monic polynomial of positive degree in one indeterminate over $A$ factors completely into monic linear factors,

(3) distinct minimal primes of $A$ are comaximal, and

(4) the set of minimal primes of $A$, Min Spec $A$, is an extremally disconnected space in the inherited Zariski topology.

\section{The result.}

THEOREM. The following conditions on a completely regular Hausdorff space $X$ are equivalent.

(a) $X$ is extremally disconnected.

(b) $C(X)[i]$ is totally integrally closed.

(c) $C^{*}(X)[i]$ is totally integrally closed.

Proof. We show (b) $\Leftrightarrow(\mathrm{c}) \Leftrightarrow(\mathrm{a})$. Let $S$ be the set of functions in $C^{*}(X)[i]$ which never vanish. It is easy to see that $C(X)[i]$ $=S^{-1} C^{*}(X)[i]$, and that $C^{*}(X)[i]$ is integrally closed in $C(X)[i]$. By [4, Propositions 1 and 7], these two rings are totally integrally closed or not alike. Now assume (c). The obvious map $X \rightarrow$ Spec $C^{*}(X)[i]$ which takes $x$ into $\{f: f(x)=0\}$ is an embedding, and

Received by the editors April 18, 1969.

1 This research was partially supported by NSF grant GP-8496. 
the image is dense. But Spec $C^{*}(X)[i]$ must be extremal [4, Proposition 3]; hence, $X$ must be extremally disconnected.

Now assume (a). We verify conditions (1)-(4) given in the introduction for $A=C^{*}(X)[i]$. $A$ is obviously reduced (no nilpotents). Condition (3) is immediate because $X$ is extremally disconnected $\Rightarrow X$ is an $F$-space $[2$, p. $214, \mathrm{~N} 4]$, and $X$ is an $F$-space $\Leftrightarrow(3)$ holds [2, p. 208]. (4) is immediate from [3, Theorem 5.3]. I wish to thank the referee for this short proof of (3) and (4). To prove (2), we first note that $X$ is zero dimensional in the sense of $[2$, p. 246]. (A space $X$ is zero dimensional iff, equivalently, either (1) disjoint zero sets in $X$ have disjoint clopen neighborhoods, or (2) the Stone-Čech compactification $\beta X$ of $X$ has a clopen basis.) $X$ is zero dimensional iff $\beta X$ is, and if $X$ is zero dimensional, so is each cozero set $U \subset X$. For if $f$ is a nonnegative function vanishing precisely on $X-U$, for each positive integer $n$, let $V_{n}$ be a clopen set in $X$ containing the zero set $f^{-1}([1 / n,+\infty])$ and disjoint from the zero set $f^{-1}([0,1 /(n+1)])$ : then $V_{n} \subset V_{n+1}$, all $n$, and $U=\bigcup_{n}\left(V_{n+1}-V_{n}\right)$ is a disjoint union of clopen subsets of the zero dimensional space $X$. We are now ready to complete the proof of the theorem by establishing

Lemma. If $X$ is zero dimensional, then every monic polynomial of positive degree in one indeterminate $t$ over $C^{*}(X)[i]$ splits into monic linear factors.

Proof. Let $F=F(t)=t^{n}+\sum_{j=0}^{n-1} h_{j} t^{j} \in C^{*}(X)[i][t]$ be monic of degree $n \geqq 1$. For each $x$ in $X$ let $F_{x}(t)=t^{n}+\sum_{j=0}^{n-1} h_{j}(x) t^{j} \in C[t]$. We use induction on $n$. The case $n=1$ is trivial. Assume that $n>1$ and that smaller degree polynomials split (for all zero dimensional $X$ ). Let $Y=\left\{y \in X\right.$ : all roots of $F_{y}$ are equal, i.e. $F_{y}=\left(t-c_{y}\right)^{n}$ for some $\left.c_{y} \in C\right\}$. $\sum_{j=0}^{n-2}\left|h_{j}-\left(\begin{array}{c}n \\ j\end{array}\right)\left(h_{n-1} / n\right)^{n-j}\right|$ vanishes precisely on $Y$. Hence $X-Y$ is a cozero set and is zero dimensional. Suppose $F \mid X-Y$ splits. Then we can extend each root function continuously (uniquely) to $X$ by giving it the value $c_{y}$ for each $y \in Y$. Continuity here follows from the Hurwitz theorem $[\mathbf{5}$, p. 4 , Theorem (1.5) $]$. Hence, we may assume without loss of generality that $Y=\varnothing$.

By considering $F\left(t-(1 / n) h_{n-1}\right)$ instead of $F$ we may also assume that $h_{n-1}=0$. Let $h=\sum_{j=0}^{n-2}\left|h_{j}\right|^{1 / n-j} \in C^{*}(X)$. Since $Y=\varnothing$, the roots of $F_{x}$ are never all zero, and hence $h$ never vanishes. Let $G=t^{n}$ $+\sum_{j=0}^{n-1} g_{j} t^{j}=h^{-n} F(h t)$, so that $g_{j}=h_{j} / h^{n-j}$ (in particular, $g_{n-1}=0$ ). An easy computation shows $\left|g_{j}\right| \leqq 1$ for each $j$, so that $G \in C^{*}(X)[i][t]$, and it is clear that if $G$ splits, so does $F$. Since $\sum_{j=0}^{n-2}\left|g_{j}\right|^{1 / n-j}=1$, we may also assume without loss of generality that $h=1$. 
We use a superscript $e$ to denote the unique continuous extension of an element of $C^{*}(X)[i]$ to $\beta X$, and also to denote the result of extending all the coefficients of a polynomial. Clearly, it suffices to show that $F^{e} \in C^{*}(\beta X)[i][t]$ splits. We still have $h_{n-1}^{e}=0$. Moreover, since $h=1, H^{e}=\sum_{j=0}^{n-2}\left|h_{j}^{e}\right|^{1 / n-j}=1$, so that the coefficients of $F^{e}$ never vanish simultaneously. Since $h_{n-1}^{e}=0$, the roots of $F_{x}^{l}$ are never all equal, for then they would all have to be zero. Thus, the hypothesis of $Y=\varnothing$ is preserved if we pass to consideration of $\beta X$ instead of $X$.

Thus, we may assume without loss of generality that $X$ is compact and that $F_{x}$ does not have $n$ equal roots at any point. It now suffices to show that each point $x \in X$ has some neighborhood $U$ such that $F \mid U$ splits over $U$, for we then get a finite cover of $X$ by clopen sets on which $F$ splits. Let $x \in X$ be arbitrary. Partition the roots of $F_{x}$ into two disjoint nonempty sets $A, B$, and choose $\epsilon>0$ less than half the distance of $A$ from $B$. For some sufficiently small open neighborhood $U$ of $x$, for each $u \in U$ there will be a corresponding partition $A(u), B(u)$ of the roots of $F_{u}$, where, counting multiplicities, $A(u)$ has the same number of elements as $A$ and $B(u)$ as $B$. This follows from the Hurwitz theorem: $A(u)$ consists of those roots within distance $\epsilon$ of some element of $A$, and similarly for $B(u)$. Let

$$
G_{u}=\prod_{a \in A(u)}(t-a)^{m(a)}
$$

and

$$
H_{u}=\prod_{b \in B(u)}(t-b)^{m(b)}
$$

where $m(c)$ is the multiplicity of $c$ as a root of $F_{u}$. It follows easily from the Hurwitz theorem that the coefficients of $G_{u}$ and $H_{u}$ depend continuously on $u$, so that $F$ factors nontrivially $F=G H$ over $U$. By the induction hypothesis, $G$ and $H$ both split over $U$.

Note that the conclusion of the lemma fails if we let $X=\mathbf{C}$ or $X=\{c \in \mathbf{C}:|c|=1\}$. Let $h$ be the inclusion map of $X$ into $\mathbf{C}$, and let $F(t)=t^{n}-h$ for any integer $n \geqq 2$. The referee has pointed out the following startling example where $X$ has a basis of clopen sets but the lemma fails: In the construction of the space $\Delta_{1}$ in $16 \mathrm{M}$ on p. 264 of [2], use the unit circle of the complex plane instead of $[0,1]$, and let $h$ be the restricted product projection from the resulting space $X$ to the circle. Then once more $F(t)=t^{n}-h, n \geqq 2$, does not split. 
AdDed In PRoOF. The lemma follows from the results of R. S. Countryman, On the characterization of compact Hausdorff $X$ for which $C(X)$ is algebraically closed, Pacific J. Math. 20 (1967), 433-448.

\section{REFERENCES}

1. E. Enochs, Totally integrally closed rings, Proc. Amer. Math. Soc. 19 (1968), 701-706. MR 37 \#199.

2. L. Gillman and M. Jerison, Rings of continuous functions, The University Series in Higher Mathematics, Van Nostrand, Princeton, N. J., 1960. MR 22 \#6994.

3. M. Henriksen and M. Jerison, The space of minimal prime ideals of a commutative ring, Trans. Amer. Math. Soc. 115 (1965), 110-130. MR 33 \#3086.

4. M. Hochster, Totally integrally closed rings and extremal spaces, Pacific J. Math. (to appear).

5. M. Marden, The geometry of the zeros of a polynomial in a complex variable, Mathematical Surveys, no. 3, Amer. Math. Soc., Providence, R. I., 1949. MR 11, 101.

University of Minnesota, Minneapolis, Minnesota 55455 